\title{
OPTICAL SOLITONS WITH HIGHER ORDER DISPERSIONS IN PARABOLIC LAW MEDIUM BY TRIAL SOLUTION APPROACH
}

\author{
Ahmed H. Arnous ${ }^{1}$, Mohammad Mirzazadeh ${ }^{2}$, Qin Zhou ${ }^{3}$, \\ Seithuti P. Moshokoa ${ }^{4}$, Anjan Biswas ${ }^{4,5}$ \& Milivoj Belic ${ }^{6}$ \\ ${ }^{1}$ Department of Engineering Mathematics and Physics, \\ Higher Institute of Engineering, El Shorouk, Egypt \\ 2 Department of Engineering Sciences, Faculty of Technology and Engineering, \\ East of Guilan, University of Guilan, P.C. 44891-63157, Rudsar-Vajargah, Iran \\ 3 School of Electronics and Information Engineering, \\ Wuhan Donghu University, Wuhan-430212, PR China \\ 4 Department of Mathematics and Statistics, \\ Tshwane University of Technology, Pretoria-0008, South Africa \\ ${ }^{5}$ Faculty of Science, Department of Mathematics, \\ King Abdulaziz University, Jeddah-21589, Saudi Arabia \\ 6 Science Program, Texas A and M University at Qatar, \\ PO Box 23874, Doha, Qatar
}

\begin{abstract}
This paper obtains bright, dark and singular soliton solutions in optical fibers with parabolic law nonlinearity in presence of third and fourth order dispersions. The trial solutions approach is employed to carry out this integration. Besides solitons, periodic singular solutions are also obtained as a byproduct. The corresponding constraint conditions are also listed.
\end{abstract}

OCIS Codes: 060.2310; 060.4510; 060.5530; 190.3270; 190.4370

(C) 2016. This manuscript version is made available under the Elsevier user license http://www.elsevier.com/open-access/userlicense/1.0/ 


\section{INTRODUCTION}

Optical solitons is one of the main topics of research in modern telecommunications industry. Several advances in this field of research, during the past few decades, led to modern day marvel in telecom systems [1-10]. Internet activity, facebook, twitter and all such communication means are the outcomes of research in solitons and other related topics in quantum and nonlinear optics. The dynamics of soliton propagation through optical fibers and other such waveguides is governed by the well known nonlinear Schrödinger's equation. It is the delicate balance between dispersion and nonlinearity that results in the formation of these solitons which are responsible for long distance information transfer.

This paper will focus on one particular type of optical fiber that appears with parabolic law nonlinearity and is also visible in sulfonate cystals. There was little or no attention given to address the propagation of optical beams in the fifth order nonlinear media. This was primarily because no exact analytical solutions were available during those days. However, recent developments have reignited acute interest in this avenue. Doped glasses with $\chi^{(5)}$ nonlinearity maintain cubic-quintic or parabolic law of nonlinearity. This paper will obtain bright, dark and singular soliton solutions to optical fibers with parabolic law nonlinearity that come with higher order dispersion in addition to the usual group velocity dispersion (GVD). The integration scheme will be trial solution approach in this paper.

\subsection{GOVERNING EQUATION}

In this section, the nonlinear dynamics of parabolic law medium with third-order dispersion (3OD), fourth-order dispersion (4OD), and self-steepening will be investigated analytically. The governing equation for the propagation of ultra-short optical solitons is given by the following NLSE $[1,10]$ :

$$
i u_{x}-\frac{\beta_{2}}{2} u_{t t}-i \beta_{3} u_{t t t}-\frac{\beta_{4}}{24} u_{t t t t}+\gamma_{1}|u|^{2} u+\gamma_{2}|u|^{4} u+i s\left(|u|^{2} u\right)_{t}=0,
$$

where the dependent variable $u(x, t)$ is the normalized electric-field envelope, while the independent variables $x$ and $t$ represent the longitudinal coordinate along the optical fibers and time coordinate in a reference frame that moves with the pulse group velocity, respectively. The first, second, third, and fourth terms in Eq. (1) represent the time evolution terms, GVD, 3OD, and 4OD, while the fifth and sixth terms account for the polynomial law nonlinearity (cubic-quintic nonlinearity) and finally the last term is due to self-steepening. We assume that the traveling wave solutions of Eq. (1) is of the form [10]

$$
u(x, t)=U(\xi) e^{i(k x-\omega t)},
$$

where $\xi=K x-v t$, and the unknown function $U(\xi)$ represents the pulse shape. Substituting this hypothesis into Eq. (1), and making some calculations yields

$$
\left(C+18 s \beta_{4} v^{2} U^{2}\right) U^{\prime \prime}+36 s \beta_{4} v^{2} U U^{\prime 2}+D U-24\left(\gamma_{1}+\omega s\right) B U^{3}-24 \gamma_{2} B U^{5}=0,
$$

and

$$
\begin{gathered}
B=6 \beta_{3}-\beta_{4} \omega \\
C=6\left(2 \beta_{2}+12 \beta_{3} \omega-\beta_{4} \omega^{2}\right) v^{2} B-\beta_{4} v\left(6 K-6 \beta_{2} \omega v-18 \beta_{3} \omega^{2} v+\beta_{4} \omega^{3} v\right), \\
D=\left(24 k-12 \beta_{2} \omega^{2}-24 \beta_{3} \omega^{3}+\beta_{4} \omega^{4}\right) B .
\end{gathered}
$$

\section{AN OVERVIEW OF TRIAL SOLUTION APPROACH}

In this section we outline the main steps of the trial equation method as follows [1]:

Step-1: We consider the following nonlinear partial differential equation for a function $u$ of two real variables, $\overline{\text { space } x}$ and time $t$ :

$$
P\left(u, u_{t}, u_{x}, u_{t t}, u_{x x}, u_{x t}, \ldots\right)=0
$$


Under the traveling wave transformation

$$
u(x, t)=u(\xi), \quad \xi=k(x-c t),
$$

where $k, c$ are constants to be determined, we reduce Eq. (7) to a nonlinear ordinary differential equation of the form:

$$
F\left(u, u^{\prime}, u^{\prime \prime}, \ldots\right)=0
$$

where the prime' denotes the derivative $\frac{d}{d \xi}$.

Step-2: Choose the trial equation

$$
\left(u^{\prime}\right)^{2}=F(u)=\sum_{l=0}^{s} a_{l} u^{l},
$$

where $s$ and $a_{l}$ are constants to be determined. Substituting Eq. (10) and other derivative terms such as $u^{\prime \prime}$ or $u^{\prime \prime \prime}$ and so on into Eq. (9) yields a polynomial $G(u)$ of $u$. According to the balance principle we can determine the value of $s$. Setting the coefficients of $G(u)$ to zero, we get a system of algebraic equations. Solving this system, we shall determine $c, k$ and values of $a_{0}, a_{1}, \ldots, a_{s}$.

Step-3: Rewrite Eq. (10) by the integral form

$$
\pm\left(\xi-\xi_{0}\right)=\int \frac{1}{\sqrt{F(u)}} d u .
$$

According to the complete discrimination system of the polynomial, we classify the roots of $\mathrm{F}(\mathrm{u})$, and solve the integral equation (11). Thus we obtain the exact solutions to Eq. (7).

\subsection{APPLICATION TO NLSE WITH 3OD AND 4OD}

The trial equation method is applied to obtain the exact solutions of the governing equation. Balancing $U^{2} U^{\prime \prime}$ with $U^{5}$ in Eq. (3), then we get $s=4$. Using the solution procedure of the trial equation method, we obtain the system of algebraic equations as follows [1]:

$$
\begin{gathered}
\frac{C a_{1}}{2}=0, \\
C a_{2}+36 s \beta_{4} v^{2} a_{0}+D=0, \\
\frac{3}{2} a_{3} C+45 s \beta_{4} v^{2} a_{1}=0, \\
2 a_{4} C+54 s \beta_{4} v^{2} a_{2}-24\left(\gamma_{1}+\omega s\right) B=0, \\
63 s \beta_{4} v^{2} a_{3}=0, \\
72 s \beta_{4} v^{2} a_{4}-24 \gamma_{2} B=0 .
\end{gathered}
$$

Solving the above system of algebraic equations, we obtain the following results:

$$
\begin{gathered}
a_{0}=\frac{-81 D s^{2} v^{4} \beta_{4}^{2}-36 B C s v^{2} \beta_{4}\left(s \omega+\gamma_{1}\right)+B C^{2} \gamma_{2}}{2916 s^{3} v^{6} \beta_{4}^{3}}, a_{1}=0, \\
a_{2}=\frac{B\left(36 s v^{2} \beta_{4}\left(s \omega+\gamma_{1}\right)-C \gamma_{2}\right)}{81 s^{2} v^{4} \beta_{4}^{2}}, a_{3}=0, a_{4}=\frac{B \gamma_{2}}{3 s v^{2} \beta_{4}} .
\end{gathered}
$$

where $\beta_{2}, \beta_{3}, \beta_{4}, \gamma_{1}, \gamma_{2}, s, v$ and $\omega$ are all real constants, $B, C$ and $D$ are given in Eqs. (4), (5) and(6). Substituting these results into Eqs. (10) and (11), we get

$$
\pm\left(\xi-\xi_{0}\right)=\int \frac{d U}{\sqrt{a_{0}+\frac{\left(36 s \beta_{4} v^{2}\left(\gamma_{1}+\omega s\right)-\gamma_{2} C\right) B}{81 s^{2} \beta_{4}^{2} v^{4}} U^{2}+\frac{\gamma_{2} B}{3 s \beta_{4} v^{2}} U^{4}}},
$$


If we set $a_{0}=0$ in Eq. (19) and integrating with respect to $U$, we obtain the following soliton solutions of Eq

$$
\begin{aligned}
u(x, t) & = \pm \sqrt{\frac{C \gamma_{2}-36 s v^{2} \beta_{4}\left(s \omega+\gamma_{1}\right)}{27 s v^{2} \beta_{4} \gamma_{2}}} \operatorname{sech}\left[\sqrt{\frac{B\left(36 s v^{2} \beta_{4}\left(s \omega+\gamma_{1}\right)-C \gamma_{2}\right)}{81 s^{2} v^{4} \beta_{4}^{2}}}\left(K x-v t-\xi_{0}\right)\right] \\
& \times e^{i(k x-\omega t)}, \\
u(x, t) & = \pm \sqrt{\frac{36 s v^{2} \beta_{4}\left(s \omega+\gamma_{1}\right)-C \gamma_{2}}{27 s v^{2} \beta_{4} \gamma_{2}}} \operatorname{csch}\left[\sqrt{\frac{B\left(36 s v^{2} \beta_{4}\left(s \omega+\gamma_{1}\right)-C \gamma_{2}\right)}{81 s^{2} v^{4} \beta_{4}^{2}}}\left(K x-v t-\xi_{0}\right)\right] \\
& \times e^{i(k x-\omega t)} .
\end{aligned}
$$

Solutions (20) and (21) are bright and singular soliton solutions respectively and they exist for

$$
36 s v^{2} \beta_{4}\left(s \omega+\gamma_{1}\right)-C \gamma_{2}>0
$$

However, if

$$
36 s v^{2} \beta_{4}\left(s \omega+\gamma_{1}\right)-C \gamma_{2}<0
$$

the singular periodic solutions are

$$
\begin{aligned}
u(x, t) & = \pm \sqrt{\frac{C \gamma_{2}-36 s v^{2} \beta_{4}\left(s \omega+\gamma_{1}\right)}{27 s v^{2} \beta_{4} \gamma_{2}}} \sec \left[\sqrt{\frac{B\left(C \gamma_{2}-36 s v^{2} \beta_{4}\left(s \omega+\gamma_{1}\right)\right)}{81 s^{2} v^{4} \beta_{4}^{2}}}\left(K x-v t-\xi_{0}\right)\right] \\
& \times e^{i(k x-\omega t)}
\end{aligned}
$$

and

$$
\begin{aligned}
u(x, t) & =\mp \sqrt{\frac{C \gamma_{2}-36 s v^{2} \beta_{4}\left(s \omega+\gamma_{1}\right)}{27 s v^{2} \beta_{4} \gamma_{2}}} \csc \left[\sqrt{\frac{B\left(C \gamma_{2}-36 s v^{2} \beta_{4}\left(s \omega+\gamma_{1}\right)\right)}{81 s^{2} v^{4} \beta_{4}^{2}}}\left(K x-v t-\xi_{0}\right)\right] \\
& \times e^{i(k x-\omega t)}
\end{aligned}
$$

where

$$
\begin{aligned}
k= & \frac{C}{24}\left(\frac{\gamma_{2} C-36 s \beta_{4} v^{2}\left(\gamma_{1}+\omega s\right)}{81 s^{2} \beta_{4}^{2} v^{4}}\right)+\frac{\beta_{2}}{2} \omega^{2}+\beta_{3} \omega^{3}-\frac{\beta_{4}}{24} \omega^{4} \\
& K=\left(\frac{2 \beta_{2}}{\beta_{4}}+\frac{12 \beta_{3}}{\beta_{4}} \omega-\omega^{2}\right) v B+\frac{81 s^{2} \beta_{4}^{2} v^{4} D-B C^{2} \gamma_{2}}{216 s v^{3} \beta_{4}^{2} B\left(\gamma_{1}+\omega s\right)}+ \\
& \beta_{2} \omega v+3 \beta_{3} \omega^{2} v-\frac{\beta_{4}}{6} \omega^{2} v
\end{aligned}
$$

Next, upon setting

$$
a_{0}=\frac{B\left(36 s v^{2} \beta_{4}\left(s \omega+\gamma_{1}\right)-C \gamma_{2}\right)^{2}}{8748 s^{3} v^{6} \beta_{4}^{3} \gamma_{2}},
$$

in Eq. (19) and integrating with respect to $U$, we obtain the following solutions to (1).

$$
\begin{aligned}
u(x, t) & = \pm \sqrt{\frac{36 s v^{2} \beta_{4}\left(s \omega+\gamma_{1}\right)-C \gamma_{2}}{54 s v^{2} \beta_{4} \gamma_{2}}} \tan \left[\sqrt{\frac{B\left(36 s v^{2} \beta_{4}\left(s \omega+\gamma_{1}\right)-C \gamma_{2}\right)}{162 s^{2} v^{4} \beta_{4}^{2}}}\left(K x-v t-\xi_{0}\right)\right] \\
& \times e^{i(k x-\omega t)}
\end{aligned}
$$




$$
\begin{aligned}
u(x, t) & =\mp \sqrt{\frac{36 s v^{2} \beta_{4}\left(s \omega+\gamma_{1}\right)-C \gamma_{2}}{54 s v^{2} \beta_{4} \gamma_{2}}} \cot \left[\sqrt{\frac{B\left(36 s v^{2} \beta_{4}\left(s \omega+\gamma_{1}\right)-C \gamma_{2}\right)}{162 s^{2} v^{4} \beta_{4}^{2}}}\left(K x-v t-\xi_{0}\right)\right] \\
& \times e^{i(k x-\omega t)}
\end{aligned}
$$

$$
\begin{aligned}
u(x, t) & = \pm \sqrt{\frac{C \gamma_{2}-36 s v^{2} \beta_{4}\left(s \omega+\gamma_{1}\right)}{54 s v^{2} \beta_{4} \gamma_{2}}} \tanh \left[\sqrt{\frac{B\left(C \gamma_{2}-36 s v^{2} \beta_{4}\left(s \omega+\gamma_{1}\right)\right)}{162 s^{2} v^{4} \beta_{4}^{2}}}\left(K x-v t-\xi_{0}\right)\right] \\
& \times e^{i(k x-\omega t)}
\end{aligned}
$$

$$
\begin{aligned}
u(x, t) & = \pm \sqrt{\frac{C \gamma_{2}-36 s v^{2} \beta_{4}\left(s \omega+\gamma_{1}\right)}{54 s v^{2} \beta_{4} \gamma_{2}}} \operatorname{coth}\left[\sqrt{\frac{B\left(C \gamma_{2}-36 s v^{2} \beta_{4}\left(s \omega+\gamma_{1}\right)\right)}{162 s^{2} v^{4} \beta_{4}^{2}}}\left(K x-v t-\xi_{0}\right)\right] \\
& \times e^{i(k x-\omega t)}
\end{aligned}
$$

where

$$
k=\frac{\omega^{2}}{2} \beta_{2}+\omega^{3} \beta_{3}-\frac{\omega^{4}}{24} \beta_{4}-\frac{2\left(s \omega+\gamma_{1}\right)^{2}}{9 \gamma_{2}}-\frac{18 C s v^{2} \beta_{4}\left(s \omega+\gamma_{1}\right) \gamma_{2}-C^{2} \gamma_{2}^{2}}{2916 s^{2} v^{4} \beta_{4}^{2} \gamma_{2}}
$$

and

$$
\begin{gathered}
K=v \omega \beta_{2}+3 v \omega^{2} \beta_{3}-\frac{1}{6} v \omega^{3} \beta_{4}+\frac{B v}{\beta_{4}}\left(2 \beta_{2}+\omega\left(12 \beta_{3}-\omega \beta_{4}\right)\right)- \\
\quad \frac{C}{9 v \beta_{4}}-\frac{C^{2} \gamma_{2}}{324 s v^{3} \beta_{4}^{2}\left(s \omega+\gamma_{1}\right)}+\frac{s v\left(16 B\left(s \omega+\gamma_{1}\right)^{2}+3 D \gamma_{2}\right)}{8 B\left(s \omega+\gamma_{1}\right) \gamma_{2}} .
\end{gathered}
$$

Here, solutions (26) and (27) are periodic singular solutions while (28) and (29) represent dark and singular soliton solutions respectively.

\section{CONCLUSIONS}

This paper studied optical solitons in presence of 3OD and 4OD with parabolic law nonlinearity. Trial solution approach yielded bright, dark and singular solitons to the governing equation along with necessary constraint conditions that guarantees the existence of such solitons. On the flip side, singular periodic solutions fall out with reverse form of the constraints. Later, these results wil be extended to the case of polynomial law nonlinearity as well as other nonlinear forms of fibers. The outcome of that research will be reported elsewhere.

\section{ACKNOWLEDGMENTS}

The third author (QZ) was funded by the National Science Foundation of Hubei Province in China under the grant number 2015CFC891. The fourth author (SPM) would like to thank the research support provided by the Department of Mathematics and Statistics at Tshwane University of Technology and the support from the South African National Foundation under Grant Number 92052 IRF1202210126. The fifth author (AB) would like to thank Tshwane University of Technology during his academic visit during 2016. The research work of fifth and sixth authors (AB \& MB) were supported by Qatar National Research Fund (QNRF) under the grant number NPRP 6-021-1-005. The authors also declare that there is no conflict of interest. 


\section{References}

[1] A. H. Arnous, M. Mirzazadeh, S. P. Moshokoa, S. Medhekar, Q. Zhou, M. F. Mahmood, A. Biswas \& M. Belic. "Solitons in optical metamaterials with trial solution approach and Backlund transform of Riccati equation". Journal and Computational and Theoretical Nanoscience. Volume 12, Number 12, 5940-5948. (2015).

[2] M. Ballav \& A. R. Chowdhury. "On a study of diffraction and dispersion-managed soliton in a cylindrical media". Progress in Electromagnetics Research. Volume 63, 3350. (2006).

[3] S. Crutcher, A. Oseo, A. Yildirim \& A. Biswas. "Oscillatory parabolic law optical spatial solitons". Journal of Optoelectronics and Advanced Materials. Volume 14, Issues 1-2, 29-40. (2012).

[4] Y. Gurefe, A. Sonmezoglu \& E. Misirli. "Application of the trial equation method for solving some nonlinear evolution equations arising in mathematical physics". Pramana Volume 77, 1023-1029. (2011).

[5] C. S. Liu. "Trial equation method to nonlinear evolution equations with rank inhomogeneous: mathematical discussions and its applications". Communications in Theoretical Physics. Volume 45, 219-223. (2006).

[6] B. Mandal \& A. R. Chowdhury. "Spatial soliton scattering in a quasi phase matched quadratic media in presence of cubic nonlinearity". Journal of Electromagnetic Waves and Applications. Volume 21, Issue 1, 12335. (2007).

[7] M. Savescu, Q. Zhou, L. Moraru, A. Biswas, S. P. Moshokoa \& M. Belic. "Singular optical solitons in birefringent nano-fibers". Optik. Volume 127, Issue 20, 8995-9000. (2016).

[8] E. Topkara, D. Milovic, A. K. Sarma, E. Zerrad \& A. Biswas. "Optical solitons with non-Kerr law nonlinearity and inter-modal dispersion with time-dependent coefficients". Communications in Nonlinear Science nd Numerical Simulation. Volume 15, Issue 9, 2320-2330. (2010).

[9] H. Triki \& A. Biswas. "Dark solitons for a generalized nonlinear Schrödinger's equation with parabolic and dual-power law nonlinearities"'. Mathematical Methods of Applied Sciences. Volume 34, Issue 8, 958-962. (2011).

[10] Q. Zhou \& Q. Zhu. "Optical solitons in medium with parabolic law nonlinearity and higher order dispersion". Waves in Random and Complex Media. Volume 25, Issue 1, 52-59. (2015). 